Annuaire suisse de politique de développement

$17 \mid 1998$

Propriété intellectuelle : quels enjeux pour les pays en développement ?

\title{
Coopération au développement : pour un vrai partenariat entre la confédération et le secteur privé
}

Laurent Guye

\section{(2) OpenEdition}

Journals

Édition électronique

URL : http://journals.openedition.org/aspd/747

DOI : 10.4000/aspd.747

ISSN : 1663-9669

Éditeur

Institut de hautes études internationales et du développement

Édition imprimée

Date de publication : 1 avril 1998

Pagination : 117-123

ISSN : 1660-5934

Référence électronique

Laurent Guye, «Coopération au développement : pour un vrai partenariat entre la confédération et le secteur privé ", Annuaire suisse de politique de développement [En ligne], 17 | 1998, mis en ligne le 22 juillet 2012, consulté le 08 septembre 2020. URL : http://journals.openedition.org/aspd/747 ; DOI : https://doi.org/10.4000/aspd.747 


\section{COOPÉRATION AU DÉVELOPPEMENT: POUR UN VRAI PARTENARIAT ENTRE LA CONFÉDÉRATION ET LE SECTEUR PRIVÉ}

LAURENT GUYE*

P ERSONNE ne conteste aujourd'hui le rôle que jouent les flux privés dans le développement. La redistribution des tâches entre secteurs public et privé qui est intervenue depuis quelques années dans les pays en développement mêmes, la stagnation du volume de l'aide publique face à l'essor des investissements privés directs ainsi que l'abandon des a priori idéologiques font que tant du côté des donateurs que de celui des récipiendaires, on insiste désormais sur les complémentarités entre flux publics et privés et l'on recherche les effets de synergie. Il n'en a pas toujours été ainsi, et c'est le mérite du Parlement suisse d'avoir érigé dès 1976 la mobilisation des ressources du secteur privé en l'un des domaines d'activité important de l'aide au développement. La loi fédérale sur la coopération internationale au développement du 19 mars 1976 stipule en effet à son article 6 que la coopération au développement peut revêtir la forme de «mesures en vue d'encourager l'engagement de ressources du secteur privé, telles que les investissements ». Les moyens par lesquels cet effet de levier a été recherché ont évolué au fil des ans, déterminant diverses formes de partenariat entre la Confédération et le secteur privé. Cet article tente d'en donner une vue d'ensemble et d'évoquer le dialogue, parfois émaillé d'attentes contradictoires et de malentendus, par lequel les acteurs ont cherché à équilibrer les intérêts publics et privés.

\section{LES CRÉDITS MIXTES: RISQUES PUBLICS ET PROFITS PRIVÉS}

Pendant près de vingt ans, les crédits mixtes ont été, quantitativement, le principal instrument de coopération au développement en collaboration avec le secteur privé. Rappelons qu'il s'agit d'un crédit combinant une part publique (un crédit concessionnel jusqu'en 1987, un don à partir de cette date) et une part privée sous la forme d'un crédit à l'exportation octroyé par un consortium de banques suisses. La combinaison de ces deux sources de financement donne un crédit remboursable sur dix ans, dont trois de différé, et dont l'élément de libéralité atteint entre 35 et $50 \%$ en fonction de la capacité d'endettement du pays récipiendaire. Mis à la disposition du gouvernement du pays partenaire, ce crédit ne peut être utilisé que pour l'importation de biens et services d'origine suisse (jusqu'à $50 \%$ de contenu étranger est admis) dans le cadre de projets de développement conformes à la fois aux priorités du pays récepteur et aux critères de la loi suisse sur la coopération au développement. C'est là qu'a surgi un premier malentendu, avec les gouvernements partenaires aussi bien qu'avec les

* Chef de la Section promotion des investissements et financements mixtes, Service de la coopération économique au développement, Office fédéral des affaires économiques extérieures, Berne. 
exportateurs suisses. L’Office fédéral des affaires économiques extérieures a géré les crédits mixtes comme un instrument de développement, tenant à s'assurer, par des missions d'évaluation ex ante menées par des consultants indépendants, que les projets proposés répondaient aux priorités de développement et que l'offre suisse était compétitive sur le plan international (c'est-à-dire capable de l'emporter sur le plan technique et commercial, sans avoir besoin du coup de pouce d'un financement concessionnel). Or, compte tenu de la pratique beaucoup plus laxiste de plusieurs de nos partenaires de l'OCDE, quelques pays récipiendaires n'ont pas caché leur impatience devant les délais supplémentaires qu'implique la volonté suisse de procéder à des évaluations indépendantes. Quant aux exportateurs suisses, leur sentiment est parfois que l'OFAEE se montre plus royaliste que le roi et ne leur donne pas les moyens de se battre à armes égales avec leurs concurrents de l'OCDE.

Quoi qu'il en soit, c'est un montant total supérieur à 2 milliards de francs, dont 803 millions correspondent à la tranche publique, qui a été engagé de 1977 à 1996 pour les financements mixtes. Les principaux pays bénéficiaires ont été, dans l'ordre, la Chine, l'Egypte, l'Indonésie et l'Inde. Quant aux secteurs d'intervention, c'est la production et la transmission d'électricité qui viennent au premier rang, suivies par les machines-outils, la minoterie et la sidérurgie. Ce n'est pas ici le lieu de faire un bilan de l'impact de ces financements. Disons seulement que la grande majorité ont atteint l'objectif fixé; le fait que les gouvernements récipiendaires assument au moins la moitié du financement a, en principe, garanti que le choix porte sur des projets qu'ils étaient déterminés à mener à bien. Les quelques cas d'échec mettent bien en évidence une limitation importante de cet instrument: le faible degré d'engagement du partenaire privé suisse. Celui-ci n'assume en fait aucun risque: la part de la Confédération n'est pas remboursable et la part bancaire est couverte par la Garantie contre les risques à l'exportation. Une fois le marché conclu, l'exportateur n'a plus d'autres obligations que celles découlant des clauses après-vente du contrat et se tourne bien naturellement vers de nouvelles affaires. Si le projet ou l'entreprise locale qui en est le porteur fait face à des difficultés (c'est particulièrement le cas des entreprises du secteur public), c'est en fin de compte l'OFAEE qui, soucieux de la durabilité de son apport, doit mettre sur pied des mesures correctives de concert avec les autorités de contrepartie.

Le caractère limité de l'engagement du secteur privé dans le cadre des financements mixtes est l'une des raisons pour lesquelles cet instrument est destiné à s'effacer au profit d'autres mesures. Deux autres facteurs de poids poussent dans la même direction.

Il s'agit en premier lieu de l'ouverture progressive de l'économie des pays en développement, de leur solvabilité croissante' et de la libéralisation de l'accès aux devises. Les opérateurs économiques ont désormais un accès beaucoup plus facile à des crédits bancaires en monnaie forte; dès lors que l'élément don n'est pas transmis à l'utilisateur final, mais gardé par le gouvernement partenaire, il n'y a plus d'incitation à recourir à une ligne de financement mixte. L'investissement de temps requis pour satisfaire les exigences administratives du donateur et du gouvernement récipiendaire joue aussi en défaveur des crédits mixtes.

1. Nous ne nous référons pas aux pays les moins avancés qui n'ont jamais été, pour la Suisse, des destinataires de financements mixtes. 
La deuxième raison a trait aux règles de conduite en matière de financement mixte adoptées en 1992 par les pays de l'OCDE. Ces règles, qui ont pris le nom d'Ensemble d'Helsinki, visent à limiter les distorsions provoquées dans les flux commerciaux par l'utilisation combinée de fonds d'aide et de crédits à l'exportation, en restreignant très fortement le champ d'application des financements mixtes. Sans entrer dans le détail de ces règles, disons qu'elles prévoient que ne sont éligibles pour des crédits mixtes que les projets dont on peut démontrer qu'ils sont commercialement non viables (leur flux de trésorerie consolidé sur dix ans doit être négatif): par exemple, des projets d'infrastructure sociale ou des investissements dans le domaine des transports publics qui ne sont en général rentables qu'au terme d'une période de vingt-cinq ans. Ces règles ont frappé de plein fouet les secteurs sur lesquels se concentraient nos financements mixtes: la génération et la transmission d'électricité, les télécommunications et les projets industriels (minoteries, fabriques textiles, etc.). C'est ainsi que le volume annuel des dépenses au titre des crédits mixtes, qui atteignait près de 80 millions de francs avant l'adoption de l'Ensemble d'Helsinki, est tombé à moins de 30 millions de francs au cours des trois dernières années. Tous nos partenaires de l'OCDE ont connu un phénomène semblable, particulièrement sensible pour des pays comme la France, l'Italie et l'Allemagne qui, dans le passé, ont soutenu beaucoup plus généreusement leur industrie d'exportation à coups de crédits mixtes que ne l'a fait la Suisse.

\section{UNE NOUVELLE POLITIQUE DE COLLABORATION AVEC LE SECTEUR PRIVÉ:LE MESSAGE DU CONSEIL FÉDÉRAL DU 29 MAI 1996}

Par son message du 29 mai 1996 relatif à «la continuation du financement et la réorientation des mesures de politique économique et commerciale au titre de la coopération au développement», le Conseil fédéral a demandé au Parlement l'ouverture d'un cinquième crédit pluriannuel d'engagement lui permettant de financer ces mesures au cours de la période 1997-2000 tout en exposant les nouvelles bases sur lesquelles il entend utiliser ces fonds. Quelques hypothèses sous-tendent ce message :

- Un nombre croissant d'entreprises - et pas seulement parmi les grandes corporations multinationales - sont amenées à développer une partie de leur production dans les pays émergents pour se rapprocher de marchés en pleine expansion et pour abaisser leurs coûts. L'alternative «exporter ou investir» est fausse: pour continuer d'exporter vers les pays en développement, l'industrie suisse doit y investir.

- La relation de partenariat à long terme qui s'établit lorsqu'un entrepreneur de la zone OCDE investit dans un pays en développement (dans le cadre d'un joint-venture, par exemple) est qualitativement supérieure - notamment sous l'angle de la durabilité (sustainability) - à celle qui se donne lors d'une simple exportation d'équipements. Le profit dépendant de la pérennité du projet, l'entrepreneur est intéressé à réaliser de manière optimale le transfert de technologie, la formation du personnel local, le soutien au niveau de la gestion, etc. 
- La collaboration entre la Confédération et le secteur privé doit être définie sur une nouvelle base fondée sur le partage des risques, ce qui veut dire le partage des pertes et des profits.

Ces prémisses expliquent la place centrale qui revient à la promotion de l'investissement dans la nouvelle stratégie. Celle-ci se matérialise par divers instruments :

- La création conjointe avec le secteur privé d'une «Société financière suisse pour le développement » (SFSD - nom provisoire) destinée à soutenir, par des prises de participation, des crédits ou des garanties, le lancement ou l'expansion de joint-ventures dans les pays en développement comme dans les pays d'Europe centrale et orientale.

- L'expansion des services de mise en relation d'affaires aidant les entreprises des pays du Sud et de l'Est ainsi que de notre pays à trouver un partenaire. Le mandat pour l'exécution de ce programme au cours de la période 19972001 a été mis au concours et remporté par une entreprise privée de consulting et d'audit.

- La participation au financement des études préparant des projets d'investissement privés. Si l'étude débouche sur une décision d'investissement, l'avance de la Confédération doit être remboursée; en cas d'échec, celle-ci est transformée en contribution à fonds perdus.

- L'encouragement à la construction et à l'exploitation de projets privés d'infrastructure (selon les modalités build-own-operate) par l'octroi de garanties contre les risques liés aux changements du cadre régulateur de tels projets.

口 Le soutien aux activités de formation du secteur privé.

- L'appui au transfert de technologies respectueuses de l'environnement.

- Le soutien à des intermédiaires financiers dans les pays en développement (fonds de capital-risque, de partenariat, de leasing, de garantie) permettant aux entrepreneurs locaux d'avoir accès à un financement à long terme et encourageant les joint-ventures.

Il est clair que ces divers instruments ne peuvent porter leurs fruits que si les conditions-cadres dans les pays en développement sont propices. Pour contribuer à créer ce climat d'accueil favorable, l'OFAEE dispose, en amont, de mesures agissant au niveau macroéconomique - les aides à la balance des paiements et les allègements de dette - attribuées dans le cadre de programmes d'ajustement soutenus par le Fonds monétaire international et la Banque mondiale $^{2}$.

La mise sur pied de ces nouveaux instruments de soutien au secteur privé est trop récente - elle est encore en cours pour certains d'entre eux - pour qu'on puisse tirer des conclusions quant à leur impact. Toutefois, l'expérience du montage même de ces instruments ainsi que les réactions enregistrées lors de leur présentation au secteur privé permettent d'ores et déjà d'identifier les potentialités de cette approche, les difficultés à surmonter et les divergences de perception avec nos partenaires du secteur privé. Elles sont synthétisées cidessous en cinq points.

2. Il n'est malheureusement pas possible de présenter ces mesures dans le cadre de cet article. 


\section{$\square$ Subsidiarité - additionnalité}

L'apport de l'OFAEE joue-t-il vraiment son rôle de déclencheur de flux privés, ce qui est l'intention, ou prend-il la place de ces flux, n'apportant en fin de compte rien de plus au pays bénéficiaire? Il n'y a pas de recette magique et universelle pour répondre à cette question cruciale. La solution doit être modulée de cas en cas (moins un pays destinataire est attractif pour le secteur privé, plus les mécanismes d'incitation doivent être généreux) et négociée avec le partenaire privé qui, logiquement, cherchera à limiter sa mise de fonds et les risques qu'il encourt.

\section{$\square$ Court terme - long terme}

Les instruments que nous proposons au secteur privé s'inscrivent dans une vision à long terme de l'évolution de l'économie internationale, qui appellera inévitablement nos entreprises à transférer une part importante de leur technologie et de la production elle-même dans les pays en développement; ce n'est qu'en jouant la carte de la globalisation que notre économie pourra préserver l'emploi. Or, beaucoup de nos partenaires privés s'accrochent encore à une logique purement exportatrice. En d'autres termes, ils veulent vendre, et pas investir, et s'intéressent dès lors beaucoup plus aux mesures traditionnelles de soutien à l'exportation (les financements mixtes, les garanties de substitution) qu'aux nouveaux instruments. A cet égard, la réduction très nette des fonds réservés pour les crédits mixtes (120 millions de francs dans le présent créditcadre contre 350 millions dans le précédent) n'a pas été bien accueillie.

\section{$\square$ Lutter à armes égales}

Notre base de départ pour formuler nos mesures de coopération sont les besoins dans les pays en développement; le critère décisif de prise de décision est l'impact de développement que peut avoir une mesure. Nos partenaires du secteur privé regardent surtout - et c'est bien naturel - les outils dont peuvent se prévaloir leurs concurrents pour emporter des marchés et invoquent cet argument pour obtenir un soutien de la Confédération ${ }^{3}$. Ce raisonnement pourrait nous entraîner sur un terrain glissant, car il est clair que plusieurs gouvernements de l'OCDE sont sensiblement plus interventionnistes et plus «généreux » dans leur soutien à leur économie d'exportation que le nôtre. Quitte à décevoir nos partenaires du secteur privé, nous ne pouvons que réaffirmer la primauté du mandat de développement périodiquement reconduit par le Parlement dans le cadre de l'approbation des crédits-cadres. Un pas a toutefois été fait dans la direction de nos exportateurs, compte tenu de la diminution très nette du budget réservé pour les financements mixtes: la création d'un fonds d'égalisation des conditions de financement. Ce nouvel instrument, dont le rôle est dissuasif, permet à une entreprise suisse qui est la plus favorable sur le plan technique et commercial, mais qui risque de perdre un appel d'offres parce qu'un concurrent est soutenu par des conditions de financement plus favorables, d'aligner son offre de financement sur celle de la concurrence. A noter que ce fonds d'égalisation poursuit également un important objectif de développement: en dissuadant nos

3. Nous nous référons ici à des entreprises individuelles, et pas aux associations représentatives de l'industrie qui ont bien compris que nos mesures, s'inscrivant dans le cadre de la loi sur la coopération au développement de 1976, ne peuvent pas être mobilisées chaque fois qu'une entreprise cherche à emporter un appel d'offres. 
partenaires de l'OCDE de mettre leur aide au service de la promotion des exportations, il contribue à élever la qualité de l'ensemble de l'aide que reçoivent les pays en développement des membres de l'OCDE.

\section{$\square$ Eviter l'arbitraire}

Le nouveau mode de collaboration avec le secteur privé que nous voulons établir implique un changement des mécanismes de passation des marchés et de prise de décision. Dans le cas des crédits mixtes, c'est le pays récipiendaire qui a la responsabilité de passer les marchés, ce qu'il est encouragé à faire au terme d'un appel d'offres international. Ce mécanisme - en principe idéal pour garantir à la fois l'égalité de chances des entreprises intéressées et le meilleur rapport qualité-prix pour le récipiendaire - ne joue pas pour les nouveaux instruments pour lesquels l'initiative et la prise de risques par le partenaire privé sont les éléments déterminants. Pour chaque instrument, l'OFAEE fixe le domaine d'activité, l'objectif poursuivi, le mécanisme par lequel il cherche à mobiliser les ressources du secteur privé ainsi que quelques critères généraux d'éligibilité. C'est ensuite aux acteurs économiques privés de saisir l'opportunité qui leur est ainsi offerte et d'investir du temps et de l'argent pour formuler un projet susceptible de bénéficier du soutien de l'OFAEE. Ce soutien sera donné dans certains cas et refusé dans d'autres, sur la base d'une analyse de la conformité du projet proposé avec l'objectif poursuivi. Il n'est pas possible, ni souhaitable, de formuler à l'avance une liste de critères précis dont le respect scrupuleux donne en quelque sorte au requérant un droit à une prestation de la Confédération. Notre politique n'est pas figée, mais va au contraire se formulant et se modifiant sur la base des expériences concrètes que nous accumulons. Or, cette liberté indispensable d'appréciation peut donner le sentiment d'arbitraire. C'est la raison pour laquelle nous voulons, du moins pendant une période initiale de maturation de nos instruments, soumettre les propositions concrètes à un petit groupe de spécialistes extérieurs à l'administration - des praticiens du secteur privé pour la plupart. La Commission consultative pour la coopération internationale au développement restera l'instance compétente de conseil au niveau des politiques et des grandes orientations programmatiques.

\section{$\square$ Faciliter plutôt que réaliser}

Le nouveau partage des rôles avec le secteur privé implique que l'initiative revienne de plus en plus à ce dernier. Comme indiqué ci-dessus, l'OFAEE trace le cadre dans lequel la collaboration peut se réaliser, mais c'est au secteur privé de proposer les projets concrets. Voilà qui n'est pas sans conséquences sur l'organisation du travail et sur nos rapports avec les gouvernements des pays bénéficiaires. Poussé à l'extrême, cet abandon de la logique planificatrice pourrait signifier que nous nous retrouvions engagés simultanément dans un trop grand nombre de pays et avec trop de lignes d'action ou d'instruments différents, ce qui serait préjudiciable à notre capacité d'accompagnement et à l'efficacité. Il n'est désormais plus possible de prendre des engagements financiers fermes avec les autorités du pays récipiendaire, puisque le volume de dépenses dépendra de la capacité des acteurs du secteur privé, de part et d'autre, de faire usage des facilités ou des mécanismes d'incitation qui leur sont offerts. 
Un compromis doit bien sûr être trouvé entre la flexibilité totale, source de dispersion, et la culture de la planification rigide qui prévaut encore dans bien des agences d'aide.

L'OFAEE s'est engagé dans un processus d'expérimentation qui est complexe, parce qu'il se fait en relation avec de nombreux partenaires, parmi le secteur privé suisse et des pays en développement, dont les intérêts partent dans de multiples directions. Par définition, une phase d'expérimentation est faite d'essais, d'erreurs et de corrections de tir. La collaboration avec le secteur privé, aussi prometteuse qu'elle puisse apparaître, n'est pas en elle-même une panacée. Les problèmes de qualité, de durabilité et d'appropriation que rencontrent tant de projets classiques de coopération se posent également, bien que dans d'autres termes, pour les projets mis en œuvre conjointement avec le secteur privé. Pour les affronter, le secteur privé a sans doute autant à apprendre des agences d'aide que celles-ci ont à tirer de l'expérience du premier. Le dialogue, condition sine qua non d'une véritable relation de partenariat, est à l'ordre du jour. 